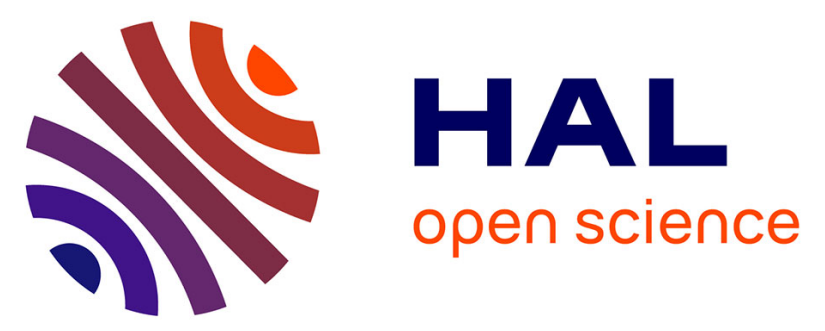

\title{
Electrical read-out of light-induced spin transition in thin film spin crossover/graphene heterostructures
}

Nikita Konstantinov, Arthur Tauzin, Ulrich Nguetchuissi Noumbé, Diana

Dragoe, Bohdan Kundys, Hicham Majjad, Arnaud Brosseau, Marc Lenertz, Aditya Singh, Stéphane Berciaud, et al.

\section{To cite this version:}

Nikita Konstantinov, Arthur Tauzin, Ulrich Nguetchuissi Noumbé, Diana Dragoe, Bohdan Kundys, et al.. Electrical read-out of light-induced spin transition in thin film spin crossover/graphene heterostructures. Journal of Materials Chemistry C, 2021, 9 (8), pp.2712-2720. 10.1039/d0tc05202g . hal-03284814

\section{HAL Id: hal-03284814 \\ https://hal.science/hal-03284814}

Submitted on 12 Jul 2021

HAL is a multi-disciplinary open access archive for the deposit and dissemination of scientific research documents, whether they are published or not. The documents may come from teaching and research institutions in France or abroad, or from public or private research centers.
L'archive ouverte pluridisciplinaire HAL, est destinée au dépôt et à la diffusion de documents scientifiques de niveau recherche, publiés ou non, émanant des établissements d'enseignement et de recherche français ou étrangers, des laboratoires publics ou privés. 


\section{Electrical Readout of Light-Induced Spin Transition in}

\section{Thin Film Spin Crossover/Graphene Heterostructure}

Nikita Konstantinov ${ }^{1}$, Arthur Tauzin ${ }^{3}$, Ulrich Nguetchuissi Noumbé ${ }^{1}$, Diana Dragoe ${ }^{3}$, Bohdan Kundys 1, Hicham Majjad ${ }^{1}$, Arnaud Brosseau 4, Marc Lenertz', Aditya Singh ${ }^{1,5}$, Stéphane Berciaud ${ }^{1}$, MarieLaure Boillot ${ }^{3}$, Bernard Doudin ${ }^{1}$, Talal Mallah ${ }^{3 *}$ and Jean-Francois Dayen 1,2*.

1. Université de Strasbourg, CNRS, Institut de Physique et Chimie des Matériaux de Strasbourg (IPCMS), UMR 7504, 23 rue du Loess, Strasbourg, 67034, France.

2. Institut Universitaire de France (IUF), 1 rue Descartes, 75231 Paris cedex 05, France.

3. Institut de Chimie Moléculaire et des Matériaux d'Orsay, Univ. Paris-Sud, Université ParisSaclay, CNRS, UMR 8182, 91405 Orsay cedex, France.

4. ENS Paris-Saclay, Université Paris-Saclay, 4 av. des Sciences, CS 30008, 91192 Gif sur Yvette, France.

5. Department of Physics, Indian Institute of Technology Delhi, 110016, New Delhi, India.

Keywords : spin crossover, graphene, molecular switches, optical device, nanoelectronics.

${ }^{*}$ To whom correspondence should be sent:

talal.mallah@universite-paris-saclay.fr,$\underline{\text { dayen@unistra.fr }}$

Abstract : Magneto-opto-electronic properties are shown for a hybrid device constructed from a spin crossover $(\mathrm{SCO})$ thin film of the $\mathrm{Fe}\left[\mathrm{HB}\left(3,5-(\mathrm{Me})_{2} \mathrm{Pz}\right)_{3}\right]_{2}$ molecular material evaporated over a graphene sensing layer. The principle of electrical detection of the light-induced spin transition in SCO/graphene heterostructures is demonstrated. The switchable spin state of the molecular film is translated into a change of conductance of the graphene channel. The low temperature write/erase process of the conductive remnant states is implemented using two distinct excitation wavelengths, in the red (LightInduced Spin State Trapping, LIESST) region for stabilizing the metastable paramagnetic state, and in the near infrared (reverse-LIESST) region for retrieving the stable diamagnetic state. The bistability of the 
system is confirmed over a significant temperature window through Light-Induced Thermal Hysteresis (LITH). This opens new avenues to the study of light-induced spin transition mechanisms exploring the coupling mechanisms between SCO system and 2D materials, providing electrical reading of the molecules/2D substrate interface. These results demonstrate how the electronic states of insulating molecular switches can be stored, read and manipulated by multiple-stimuli while transducing them into low impedance signal thanks to two-dimensional detectors, revealing the full potential of mixeddimensional heterostructures for molecular electronics and spintronics. 
Magnetic switchable molecular materials have garnered increasing attention as building blocks for data storage, imaging, photodetection, energy harvesting and thermal applications. ${ }^{[1-10]}$ They allow for the manipulation through external stimuli of multiple electronic states encoded in their switchable molecular configurations, and represent an elegant and versatile approach to store and manipulate spin information. ${ }^{[11,12]}$ The SCO complexes and networks are at the forefront capable of exhibiting reversible switching between the diamagnetic low spin (LS, S =0, ${ }^{1} \mathrm{~A}_{1 \mathrm{~g}}$ state) and the paramagnetic high spin (HS, $\mathrm{S}=2,{ }^{5} \mathrm{~T}_{2 \mathrm{~g}}$ state) states upon application of pressure, temperature, light, or electric fields. ${ }^{[2,3,13]}$ Several reports have already explored the use of SCO materials as memory and light-emitting devices, sensors, or photodetectors (for recent reviews, see for example ref. ${ }^{[3,13]}$ ).

The intrinsic very high resistivity of molecular materials remains a key bottleneck for their implementation into electronic devices. ${ }^{[14-17]}$ Hence, to circumvent the intrinsic dielectric nature of SCO systems and to be able to detect electrically the switching behavior, different strategies were implemented by combining SCO with other materials. ${ }^{[18-23]}$ However, this type of approach requires chemical engineering to preserve the switching properties of the SCO compounds. ${ }^{24]}$ An alternative elegant approach to detect the SCO state consists in using atomically-flat van der Waals two dimensional (2D) materials with their versatile electronic properties and exacerbated surface sensitivity, ${ }^{[25,26]}$ as it has been carried out for other systems. ${ }^{[27,28,37-39,29-36]}$ Among functional 2D materials, graphene is the most attractive owing to its conductance sensitivity to the environment dielectric properties. ${ }^{[40]}$ Recently, Dugay et al. ${ }^{[41]}$ pioneered the use of a graphene channel buried under a film made of SCO nanoparticles, capable to transduce electrically the thermal spin state switching. Similarly, van Geest et al. have recently shown that the thermal spin transition of a SCO single crystal can be electrically detected by a graphene channel separated by an insulating polymer spacer having a thickness of several nanometers. ${ }^{[42]}$

SCO materials are unique among magnetic molecular systems because their two-states levels can not only be manipulated by temperature but also by light, albeit at low temperatures. ${ }^{[43]}$ Photoconduction was observed in SCO based devices, ${ }^{[44,45]}$ but was not attributed to a spin transition. Indications of resistance change under illumination were reported for vertical large-area tunnel junctions made of SCO ultra-thin 
layers, ${ }^{[46]}$ however the magnitude of the response was found to be limited by their intrinsic high resistivity and possible mechanical effects. The electrical read out of the light induced spin switch has not been, to the best of our knowledge, evidenced to date.

We report here the electrical readout of the light-induced spin transition of a nano-crystalline thin film made $\mathrm{SCO}$ molecules $\left(\mathrm{Fe}\left[\mathrm{HB}\left(3,5-(\mathrm{Me})_{2} \mathrm{Pz}\right)_{3}\right]_{2}\right)$ assembled by sublimation under vacuum (Figure 1a) on the top of a graphene detector. We first evidence that the device electrically detects the thermal SCO transition, then we demonstrate, for the first time, the electrical detection of the LIESST, reverseLIESST, ${ }^{[43]}$ and LITH $^{[47-49]}$ of the thin film. This is unambiguously confirmed by matching the lightinduced electrical signatures of the device with the optical measurements on a thin film with similar characteristics. It is important to note that thin films obtained by vacuum-evaporation of a SCO molecular material, distinct from layers of nanometer-sized particles and form bulkier crystals, offer advantages in terms of scalability and compatibility with physical processes used for devices fabrication, circumventing the use of solvent and making possible clean interface with expected optimum coupling with graphene.[3,50,51]

\section{RESULTS AND DISCUSSION}

We first design the conducting channels made of $300 \mu \mathrm{m}$ wide and $1.5 \mathrm{~mm}$ long stripes of a monolayer of graphene on $\mathrm{SiO}_{2}$ (see Figure $1 \mathrm{~b}$ and Methods for the detail of fabrication). The $\mathrm{SCO}$ molecules $\left(\mathrm{Fe}\left[\mathrm{HB}\left(3,5-(\mathrm{Me})_{2} \mathrm{Pz}\right)_{3}\right]_{2}\right.$, inset of Figure 1a) are then evaporated in parallel over the graphene channels and over a non-patterned graphene/ $/ \mathrm{SiO}_{2}$ substrate as previously described. ${ }^{[52]}$ Raman spectra have been performed on bare graphene area and on $\mathrm{SCO} /$ graphene region on the same device, showing that SCO molecules on graphene leads to slightly larger hole doping as compared to the bare graphene reference (see Figure S.1. in Supporting Information).

A schematic view of the final SCO/graphene heterostructures based device is depicted in Figure 1a. The graphene transistor is buried below the SCO thin film, ensuring a direct interfacial coupling between the 
two materials. The planar configuration of the device allows for direct top access to address optically the SCO layer, together with a three terminals control on the graphene conductivity.

Atomic Force Microscopy (AFM) imaging (20x20 $\mu^{2}$ image, Figure 1c) of the non-patterned substrate consists in extended, dense and oriented packing of objects with apparent thickness of $\sim 120 \mathrm{~nm}$. A zoom on a small area $\left(2 \times 2 \mu \mathrm{m}^{2}\right)$ reveals that the objects have a platelet shape with apparent large aspect ratio width/thickness of 400/20 (Figure S2a). The flat shape of the objects and their orientation revealed by the AFM images result in a very good surface covering of the graphene leading to an optimum $\mathrm{SCO} /$ graphene interface sensitivity. The thickness of the film measured by profilometry reveals an average value close to $120 \mathrm{~nm}$ with some rugosity (Figure S2b) confirming the results of AFM. In order to check crystallinity of the film, the X-ray diffraction pattern recorded in the $\theta-2 \theta$ mode (Figure S3) shows three relatively intense reflections $\left(2 \theta=10.07^{\circ}, \sim 20.3^{\circ}\right.$ and $\left.\sim 41.08^{\circ}\right)$ assignable to the same family of planes of the triclinic phase of the bulk material. The thin film consists therefore of densely-packed nanocrystals as suggested by AFM and a preferred orientation is evidenced with the (100) or (01-1) plane being parallel to the surface of the film. The X-ray Photoelectron Spectroscopy (XPS) survey spectrum of the film (Figure S4a) shows the presence of all the elements characteristics of the molecule (Fe, N, C and B). The spectra recorded in the narrow energy regions at the Fe-2p, N-1s and B-1s edges (Figure S4b) on three different spots allows us to determine the average N/Fe and $\mathrm{B} / \mathrm{Fe}$ ratio of 12.4 and 2.5 , very close to the theoretical values (12 and 2, respectively). These data are consistent with those previously reported for a $130 \mathrm{~nm}$-thick film on a Si substrate, ${ }^{[52]}$ confirming the integrity of the molecules after sublimation on the device. 


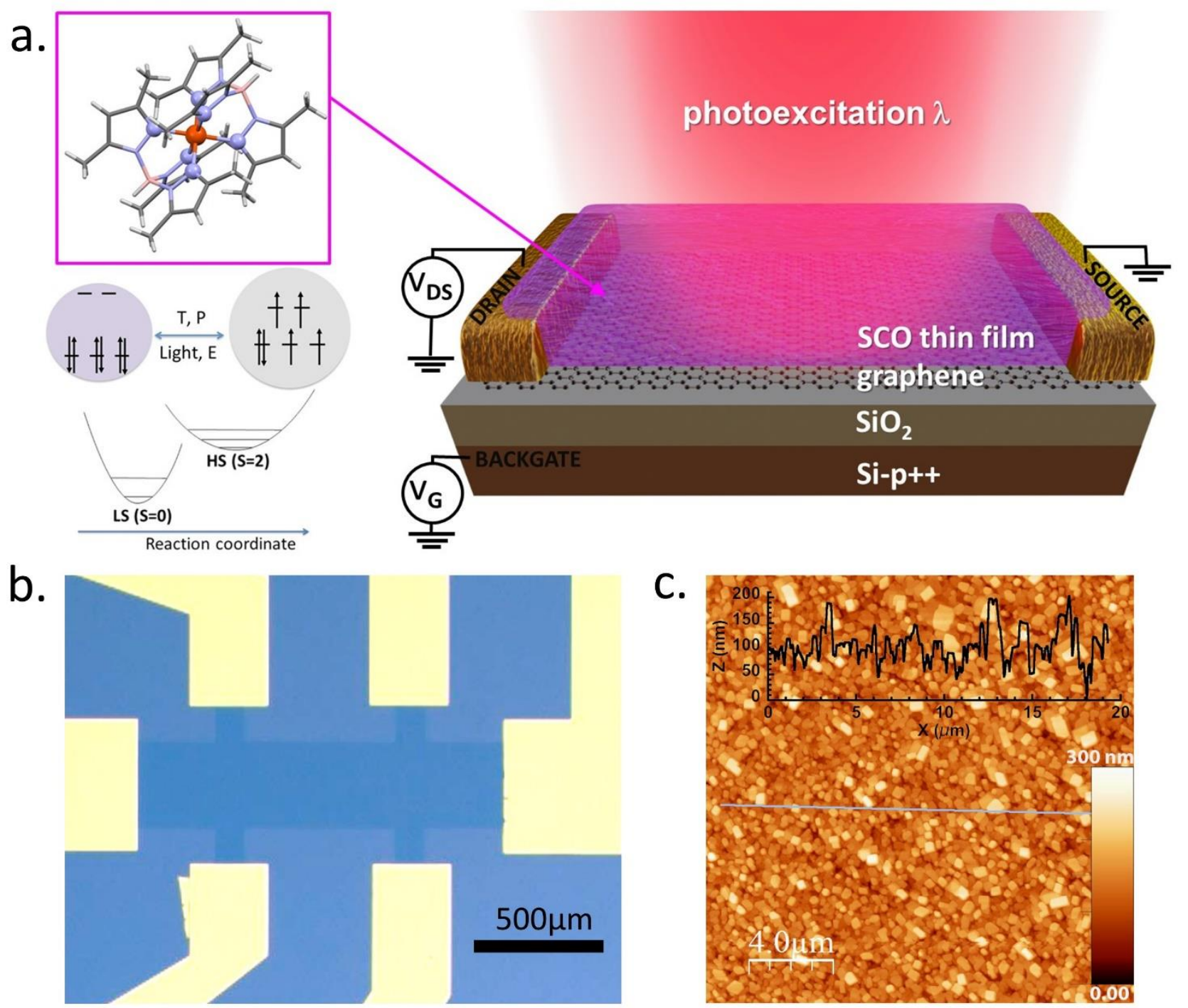

Figure 1. Thin Film Spin-crossover/Graphene Heterostructure. a. Scheme of the final optoelectronic device with the graphene sensor covered by the vacuum-sublimated SCO thin film made from $\mathrm{Fe}\left[\mathrm{HB}\left(3,5-(\mathrm{Me})_{2} \mathrm{Pz}\right)_{3}\right]_{2}$ molecules (represented in inset). Third terminal back gate control is possible through the $\mathrm{SiO}_{2}(300 \mathrm{~nm}) / \mathrm{Si}$-p ++ substrate, while direct top access allows optical addressing of the SCO active layer. The orbital energy diagram illustrates the LS (diamagnetic) and the HS (paramagnetic) states of the SCO material b. Optical microscope image of the patterned graphene sensor contacted by metallic Ti/Au electrodes. c. Topographic AFM image (20x20 $\left.\mu^{2}\right)^{2}$ with a profile showing nano-objects with a $120 \mathrm{~nm}$ average height and peaks up to $280 \mathrm{~nm}$.

Wire-bonded devices are mounted on a dip-stick inside a He cryostat fitted with an optical fiber for temperature dependent optoelectronic studies. Conductance vs. temperature study (sweep rate fixed at 0.3 K. min $^{-1}$ for all measurements) reveals a clear signature of the thermally-induced spin transition (Figure 2a shows typical behavior of our devices, see orbital energy diagram in Figure 1a). A well-marked 
hysteresis is observed, with transitions temperatures (corresponding to a fraction of HS SCO species, $\mathrm{x}_{\mathrm{HS}}$ $=0.5$ ) best estimated here by using the derivative plot dG/dT (upper panel of Figure $2 \mathrm{a}$ ), at $\mathrm{T} \downarrow=136 \mathrm{~K}$ in the cooling mode and at $\mathrm{T} \uparrow=156 \mathrm{~K}$ in the heating mode $(\Delta \mathrm{T}=20 \mathrm{~K})$. The reproducibility of the electrical response is demonstrated by measuring the conductance vs. temperature traces several times on different devices made from the same SCO/Graphene sample (average values $\mathrm{T} \downarrow=136 \pm 3 \mathrm{~K}$ and $\mathrm{T} \uparrow=$ $153 \pm 3 \mathrm{~K}, \Delta \mathrm{T}=17 \pm 6 \mathrm{~K})$ (Figure S5). The thermal switching characteristics of the film are in relatively good agreement with those of the reference $130 \mathrm{~nm}$-thick SCO film extracted from optical measurements (Figure $2 \mathrm{~b}, \mathrm{~T} \downarrow=144 \mathrm{~K}, \mathrm{~T} \uparrow=167 \mathrm{~K}, \Delta \mathrm{T}=23 \mathrm{~K}$ ), ${ }^{[51,52]}$ with small changes in the determination of transition temperatures that can be related to the differences in experimental techniques as discussed in [53]. Check measurements show the absence of both hysteresis and thermal transition of the bare graphene/SiO 2 device (Figure S6, Supporting Information), and successive heating-cooling loops (Figure S8, Supporting Information) asset of the reproducibility and stability of the device.
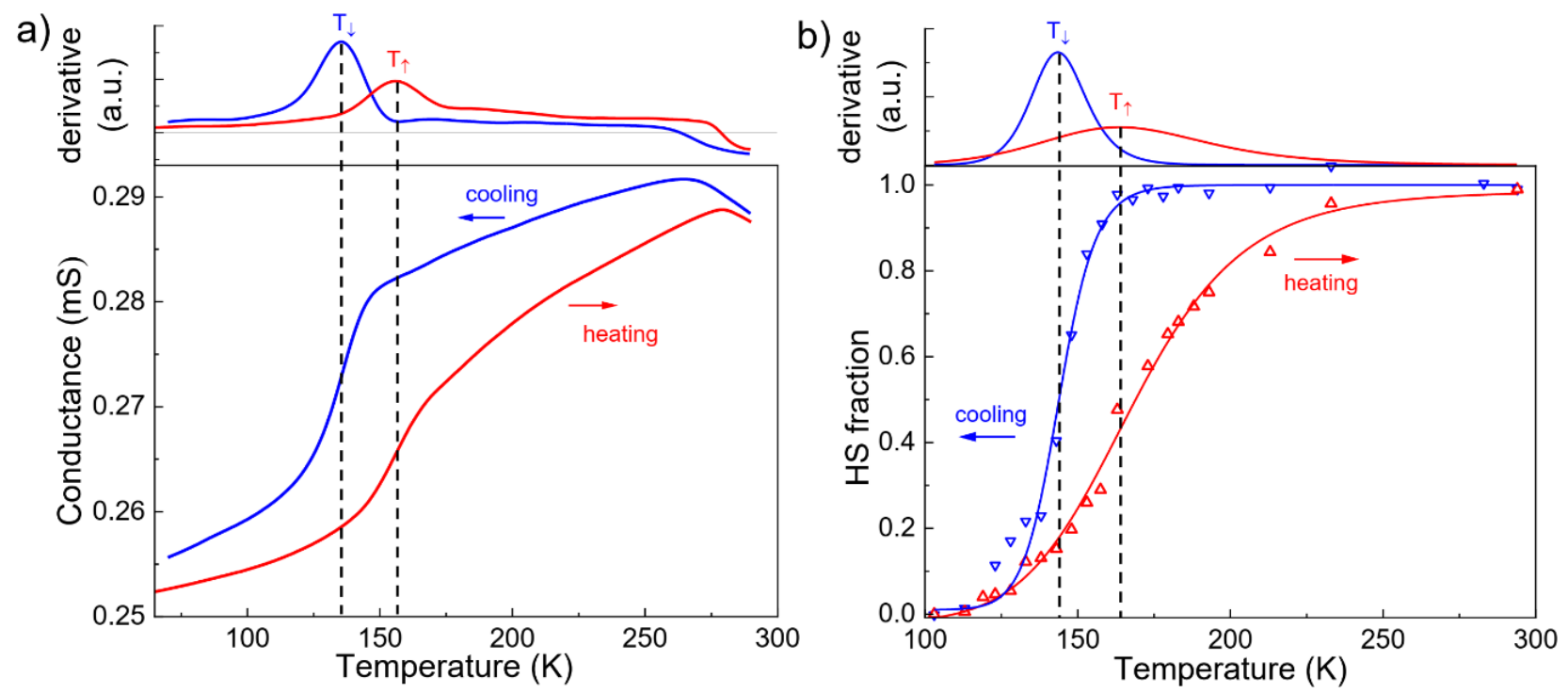

Figure 2. Thermal Spin Transition. a. Temperature dependence of the conductance of the SCO/graphene thin film heterostructure based device. Thermal transition in heating and cooling modes together with hysteresis are observed, resulting from electrical detection by the graphene sensor of the thermal spin transition of the SCO top layer. The upper panel is the derivative of the conductance vs. temperature plot, allowing to estimate precisely the transition temperatures of the cooling mode $T \downarrow=$ $136 \mathrm{~K}$ and of the heating mode T个=156 K. b. Temperature dependence of the HS fraction as extracted from $U V$-vis spectra collected for a reference $S C O$ thin film of $\mathrm{Fe}\left[\mathrm{HB}\left(3,5-(\mathrm{Me})_{2} \mathrm{Pz}\right)_{3}\right]_{2}$ of $130 \mathrm{~nm}$ 
thickness. The derivative of this plot is shown in the upper panel, and allows to estimate the transition temperatures of the cooling mode $T \downarrow=144 \mathrm{~K}$ and of the heating mode $T \uparrow=167 \mathrm{~K}$.

We then investigate the electrical response of the device upon light irradiation using well-documented experimental procedures for magnetic detection of LIESST, reverse-LIESST and LITH. ${ }^{[54]}$ We use the possibility to trap, at temperatures well below the thermal transition, the metastable paramagnetic high spin (HS) state formed by Vis excitation (Metal-to-Ligand -Charge-Transfer or the d-d bands) of the diamagnetic low spin (LS) species (LIESST effect), or to promote the reverse process (reverse-LIESST) by NIR excitation (d-d band absorption) of the HS species, ${ }^{[43]}$ as depicted inset of Figure 3a. The LIESST experiments are performed at $10 \mathrm{~K}$ (Figure 3), following the same protocol reported by Zhang et al.[55] We photo-excite the SCO thin film in the red light range $\left(\lambda_{1}=647 \mathrm{~nm}\right.$, power $550 \mu \mathrm{W} / \mathrm{mm}^{2},{ }^{1} \mathrm{~A}_{1 \mathrm{~g}} \rightarrow{ }^{1} \mathrm{~T}_{1 \mathrm{~g}}$ transition) or higher, to keep a photon energy smaller than the energy of the trap states at the silica surface and prevent photogating spurious signal at the graphene/SiO ${ }_{2}$ interface. ${ }^{[56,57]}$ Series of irradiation/relaxation steps of 15 minutes each at $10 \mathrm{~K}$ were carried out to test the possible occurrence of background signal not related to SCO. A reversible small change of the resistance observed (Figure S7) when switching the light on and off is attributed to photo-thermoelectric effects at the Ti/Au contacts, also observed on graphene-only devices. ${ }^{[58]}$ In addition, we observe that the optical excitation of the SCO thin film induces a main remnant increase of the graphene channel conductance (not present with the bare graphene device, see Figure S7) that starts saturating after approximately two hours of accumulated irradiation time (Figure 3 and Figure S7). This pinpoints the LIESST effect as being responsible for the remnant conductance change of the graphene. The transduction mechanism is expected to have the same origin as for the thermal spin transition, and is presumably related to the SCO transition following a nucleation-growth dynamics at the macroscopic bulk-like length scale expected for the thin film prepared here. ${ }^{[59]}$

The reverse-LIESST experiments are performed by illuminating the device with near-infrared light $\left(\lambda_{2}\right.$ $=820 \mathrm{~nm}, 100 \mu \mathrm{W} \cdot \mathrm{mm}^{-2},{ }^{5} \mathrm{~T}_{2 \mathrm{~g}} \rightarrow{ }^{5} \mathrm{E}_{\mathrm{g}}$ transition). We observe an inverse change of the graphene conductance compared to the LIESST effect (Figure 3a), which indicates that a fraction of the HS species 
has been successfully reconverted into the LS state. Comparison with magnetic measurements (Figure 3b) confirms that the evolution of the sample conductance matches the magnetic response of the SCO material. Note that the partial HS to LS photoconversion observed both in the conductance (temperature) trace of the $\mathrm{SCO} /$ graphene heterostructures and in the photo-magnetic signal (respectively Figure $3 \mathrm{a}$ and Figure $3 \mathrm{~b}$ ) is typical for the photo-stationary state resulting from the spectral overlap of the ${ }^{5} \mathrm{~T}_{2 \mathrm{~g}} \rightarrow{ }^{5} \mathrm{E}_{\mathrm{g}}$ and the ${ }^{1} \mathrm{~A}_{1 \mathrm{~g}} \rightarrow{ }^{3} \mathrm{~T}_{1 \mathrm{~g}, 2 \mathrm{~g}}$ absorption bands, as established in the case of bulk sample. ${ }^{[43]}$ The difference in the photoexcitation dynamics between LIESST measurements performed on $\mathrm{Gr} / \mathrm{SCO}$ heterostructure (Figure 3.a) and on SCO materials (Figure 3.b) mainly results from the distinct experimental techniques, together with the incoming specific light intensities and sample thicknesses which are not the same in the two systems.
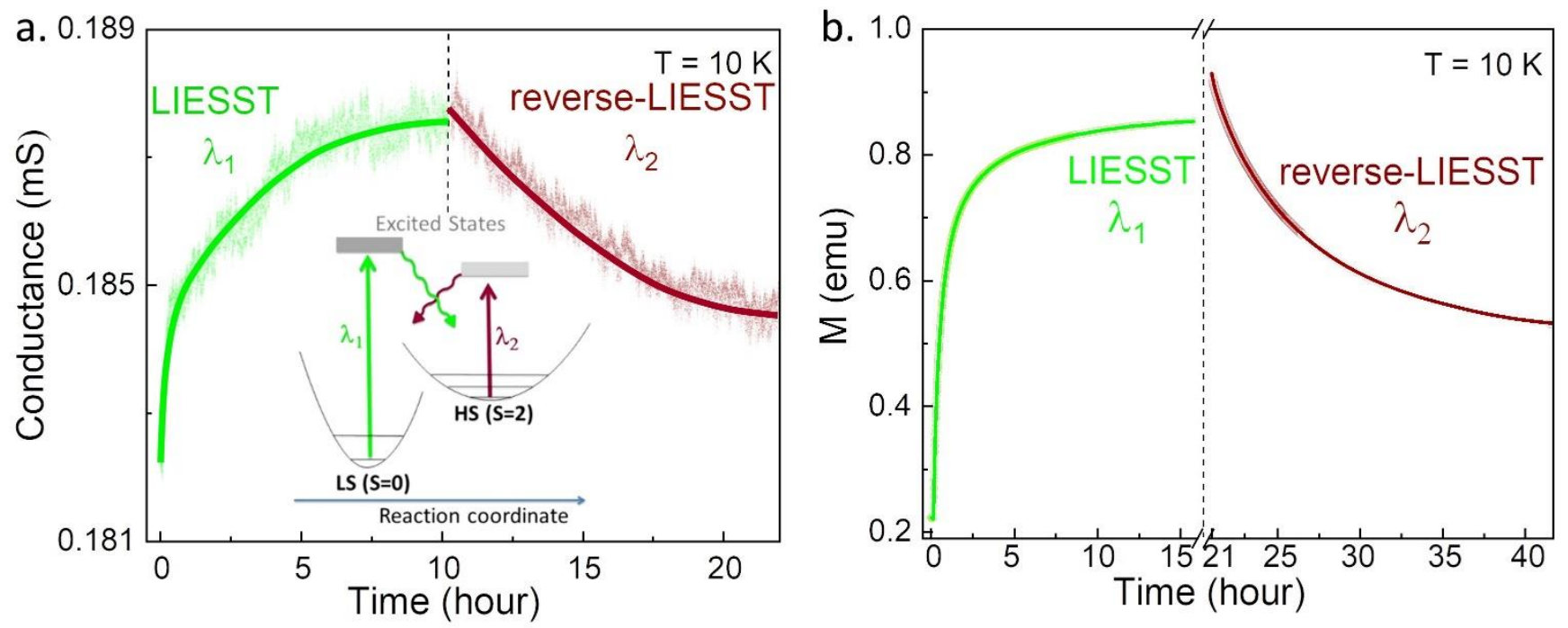

Figure 3. Light-Induced Spin Transition. a. Conductance vs. time plots of SCO/graphene heterostructure device under continuous red light excitation $\left(\lambda_{1}=647 \mathrm{~nm}, 550 \mu \mathrm{W} . \mathrm{mm}^{-2}\right)$ during 10 hours demonstrating the LIESST effect, followed by excitation under near-IR light $\left(\lambda_{2}=820 \mathrm{~nm}, 100 \mu \mathrm{W} . \mathrm{mm}^{-2}\right)$ showing the reverse-LIESST effect. Inset: Energy band diagram describing the photoexcitation spin transition mechanisms. In green (respectively in brown), the LIESST effect (respectively, reverse-LIESST effect) results from photoexcitation of SCO molecule from the diamagnetic $L S$ state (resp. paramagnetic HS state) to excited states under red light (resp. Near-IR) excitation of wavelength $\lambda_{1}$ (resp. $\lambda_{2}$ ), before relaxing towards the HS state (resp. LS state). b. Magnetization vs time plots showing the photomagnetic signal recorded at $10 \mathrm{~K}$ upon successive irradiation of the bulk sample with $\lambda_{1}=635 \mathrm{~nm}$ and $\lambda_{2}=850$ nm as in ref. [55] 
Temperature studies of the LS $\leftrightarrow \mathrm{HS}$ transitions are performed to confirm our interpretation of electrical detection of LIESST and reverse-LIESST. The sample is brought to the LS state by cooling from ca. 200 $\mathrm{K}$ down to $10 \mathrm{~K}$ and the conductance vs. temperature is recorded (blue curve, Figure $4 \mathrm{a}$ ). Then, it is irradiated with red light $\left(\lambda_{1}=647 \mathrm{~nm}, 550 \mu \mathrm{W} \cdot \mathrm{mm}^{-2}\right)$ during one hour. The change in the conductance of the device is the signature of the population of the metastable HS state. Upon heating (red curve, Figure 4a), the conductance of the device remains almost constant up to $\mathrm{T}=35 \mathrm{~K}$, then decreases and reaches at $\mathrm{T}=60 \mathrm{~K}$ its original value during the cooling process following a thermally activated process as for the classical magnetic response of the relaxation after the LIESST effect. ${ }^{[54,60]}$ The relaxation temperature ( $\mathrm{T}_{\text {LIESST }}$ ) above which the metastable HS state is erased can be estimated (see upper panel in Figure $4 \mathrm{~b}$ ) from the extremum of the derivative curve $\mathrm{dG} / \mathrm{dT}$ at $\mathrm{T}_{\text {LIESST }}=48.5 \mathrm{~K}$ (following a similar determination method than for magnetic susceptibility measurements where $T_{\text {LIEEST }}$ is extracted from the derivative $\mathrm{d} \chi_{\mathrm{M}} / \mathrm{dT} \cdot{ }^{[54,61]}$ ). While heating the sample further, the thermal spin transition is recovered at the transition temperature $\mathrm{T} \uparrow$ (Figure 4a). The conductance vs temperature study allows us to get a rough estimate of the fraction of SCO molecules having switched from the LS state to the HS state upon light excitation. While comparing the amplitude of change of the conductance value at $10 \mathrm{~K}$ before and after irradiation, to the amplitude of change upon thermal spin transition which corresponds to a complete transition from $\mathrm{x}_{\mathrm{HS}}=0$ to $\mathrm{x}_{\mathrm{HS}}=1$, we can conclude that after 1 hour of light excitation at $10 \mathrm{~K}$, roughly $75 \%$ of the SCO population get switched into the HS state.

Temperature dependent studies allow us to provide evidence of the light-induced bistability in our $\mathrm{SCO} /$ graphene heterostructure and its electrical readout. To do so, we performed temperature dependent studies of the conductance of the heterostructure under continuous red light excitation (Figure 4c, 0.3 $\mathrm{K} . \mathrm{min}^{-1}$ thermal sweep rate, $550 \mu \mathrm{W} / \mathrm{mm}^{2}$ ) that reveals a thermal hysteresis in the 10 - $60 \mathrm{~K}$ range absent for LIESST (Figure 4b) and consistent with the LITH process observed on bulk materials using a magnetic readout. ${ }^{[47-49]}$ The bistability induced by light is due to the direct competition between the LS $\rightarrow$ HS molecular photoexcitation and the HS $\rightarrow$ LS self-accelerated relaxation characterizing the cooperative 
macroscopic system. The curve observed upon heating under light excitation (Figure 4c) differs from the one measured in the dark after LIESST excitation (Figure 4b) mainly because of the presence of an intermediate kinetic step between 30 and $40 \mathrm{~K}$ when the thermal relaxation takes over. Near the $\mathrm{T}_{\text {LIESST }}$ value, compared to measurement in the dark, the signal variation becomes sharper before the slowing down due to the recovery of the LS state. The branches of the hysteresis loop observed in the heating and the cooling modes are respectively indicative of the actuation and the end of the thermal relaxation regime of the photoexcited states. Interestingly, the electrical signature of the LITH process in our heterostructures draws a remarkably well-shaped hysteresis loop $\left(\mathrm{T}_{\text {down }} \approx 7 \mathrm{~K}, \mathrm{~T}_{\text {up }}=52 \mathrm{~K}\right.$ and $\Delta \mathrm{T} \approx 45 \mathrm{~K}$, estimated from the derivative curves $\mathrm{dG} / \mathrm{dt}$ in the upper panel of Figure 4c). This particular shape of hysteresis is ascribed to the, a priori, homogeneous excitation of the thin film as the thickness is much lower than the penetration depth of light. With this respect, the observation of the smooth evolution preceding the steeper one in the two branches of the hysteresis loop might be a consequence of the large distribution of the size of the nanocrystals forming the film (AFM images Figure 1c and Figure S2) and thus, the convolution of a slow non-cooperative relaxation (random spin-mixing, small-enough nanocrystals) with the cooperative one (clusters). ${ }^{[59,62]}$ Further studies are required for characterizing the dynamics, ${ }^{[63]}$ and its size-dependence, ${ }^{[59,62]}$ for evaluating the role of the interface of the SCO layer, ${ }^{[42,64]}$ and within the layer, the inter nanocrystals effect. 
a.

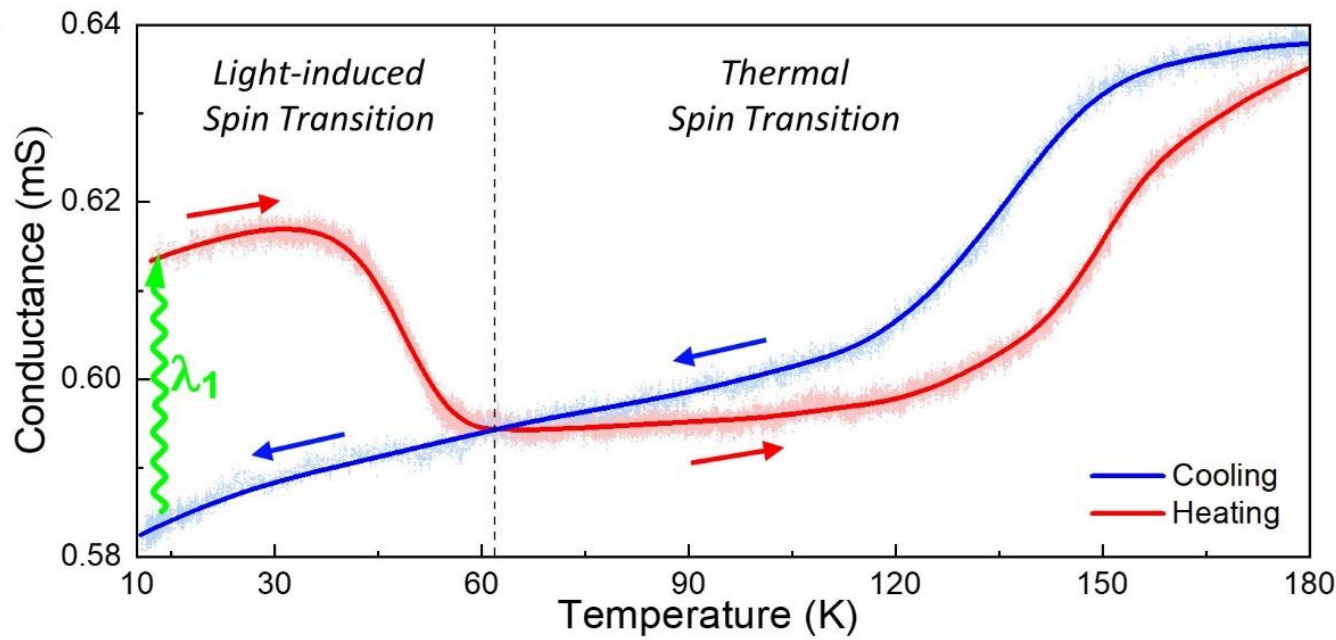

b.

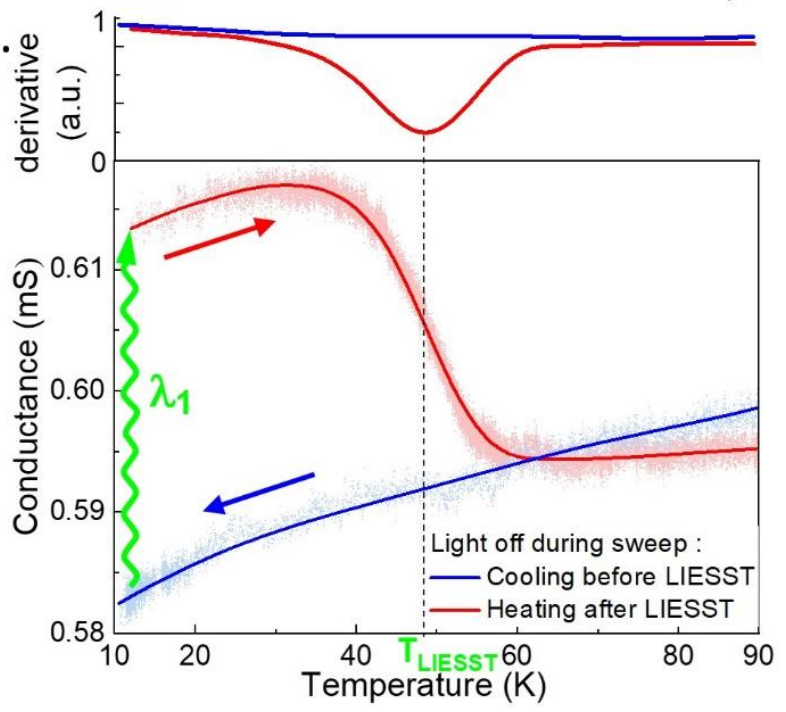

c.

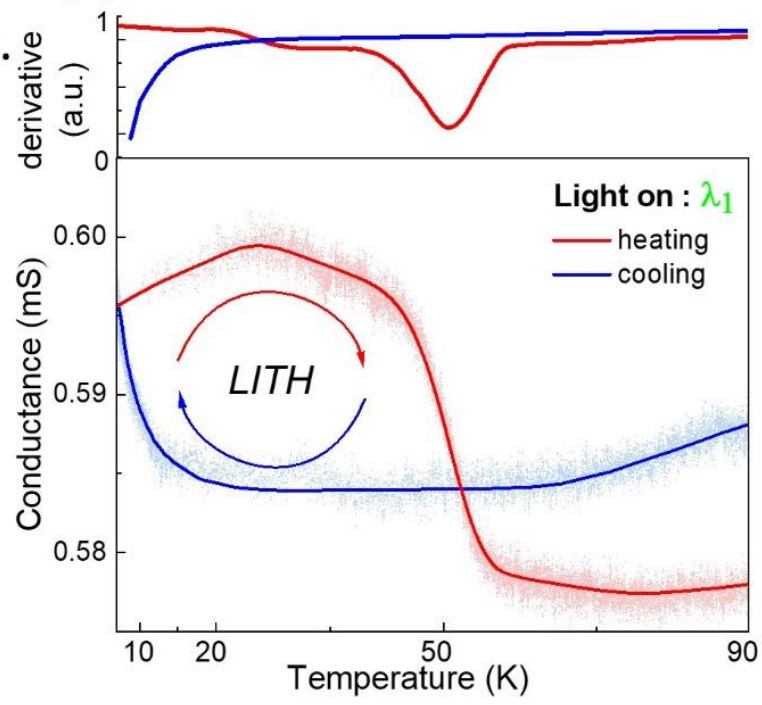

Figure 4. Temperature dependent study and bistability. a. Conductance vs temperature plots of the SCO/graphene device in the heating (red) and cooling (blue) modes. Red light photoexcitation $\left(\lambda_{1}=\right.$ $647 \mathrm{~nm}, 550 \mu \mathrm{W} . \mathrm{mm}^{-2}$ ) is performed during one hour at $10 \mathrm{~K}$ after tracing the cooling curve (in dark), before measuring in the heating mode under dark condition. The thermal spin transition phenomenon is observed at high temperatures, while below $60 \mathrm{~K}$ the Light induced spin transition appears. b. Details of the LIESST effect at low temperature. Upper panel is the derivative of the conductance vs. temperature plots, allowing to estimate the LIESST transition temperature at $T_{\text {LIESST }}=48.5 \mathrm{~K} . \boldsymbol{c}$. Conductance vs. temperature plots of the SCO/graphene device performed under continuous red-light excitation $\left(\lambda_{1}=\right.$ 647nm, $550 \mu \mathrm{W} . \mathrm{mm}^{-2}$ ). A Light Induced Thermal Hysteresis (LITH) is observed on the temperature dependence of photoconductance, indicative of the bistability of the SCO top layer.

The thermal and light induced SCO described and discussed above demonstrate the ability of the device to sense the macroscopic changes of the film despite the mere interfacial contact with graphene. It is accepted that upon variation of the electronic and chemical environment of graphene, the transfer curve 
may experience a lateral shift usually corresponding to the modulation of its doping level, or/and to a change of its slope indicative of a change of mobility and related scattering mechanisms. ${ }^{[41,65-67]}$ To get insight into the transduction mechanism responsible of the SCO detection, we carry out two series of transconductance measurements implementing the thermo- and photo-induced spin-transition processes. Current vs back gate voltage (VG) transfer curves (Figure 5a) measured on the $\mathrm{SCO} /$ graphene device during cooling in the HS state (at 160K) and then in the LS state (at $10 \mathrm{~K}$ and $100 \mathrm{~K}$ ) show the typical linear dispersion law of graphene, with a Dirac cone out of the applied back gate voltage range, indicating graphene is hole doped. The transfer curve in the HS state is shifted to the right compared to the LS state, i.e. towards higher $\mathrm{Vg}$ values, indicating an increased hole doping upon the transition from the LS to the HS state (see also schematic of the graphene band diagram for SCO compounds in both states, inset of Figure 5.b). In order to decorrelate the role of the temperature from the SCO transition, we record the transfer curves of the graphene sensor at $10 \mathrm{~K}$ before and after LIESST (Figure 5b). Upon LS $\rightarrow \mathrm{HS}$ transition, we observe a non-negligible lateral shift to the right $\left(\Delta \mathrm{V}_{\mathrm{g}} \approx+4.8 \mathrm{eV}\right)$ of the transfer curve as for the thermal transition, without noticeable change of its slope. This shift may be transduced electrostatically by the change of the doping level of graphene by the amount of charge $\Delta \mathrm{n}=\mathrm{C}_{\mathrm{SiO} 2} * \Delta \mathrm{V}_{\mathrm{g}} / \mathrm{e}$ $\approx 3.6^{*} 10^{11}$, e is the electron charge and $\mathrm{C}_{\mathrm{SiO} 2}\left(\approx 12 \mathrm{nF} \cdot \mathrm{cm}^{-2}\right)$ is the capacitance of the $300 \mathrm{~nm} \mathrm{SiO}_{2}$ layer. This estimation is to be considered carefully as, because of the strong hole doping level induced by the SCO material into graphene, the neutrality point of graphene remains out of back gate voltage experimental window. These studies pinpoints that the origin of the conductance change upon the SCO transition is a change of the hole doping level of graphene. This modification results probably from the variation of the dielectric permittivity of the film upon the spin crossover, ${ }^{[68,69]}$ inducing a modification of the electrostatic environment of graphene and, therefore, its doping level. Further insight into these mechanisms could benefit from dedicated Raman spectroscopy studies to bring complementary insight into how doping and maybe also how strain effects are contributing to the properties of such mixeddimensional heterostructure. ${ }^{[70]}$ 

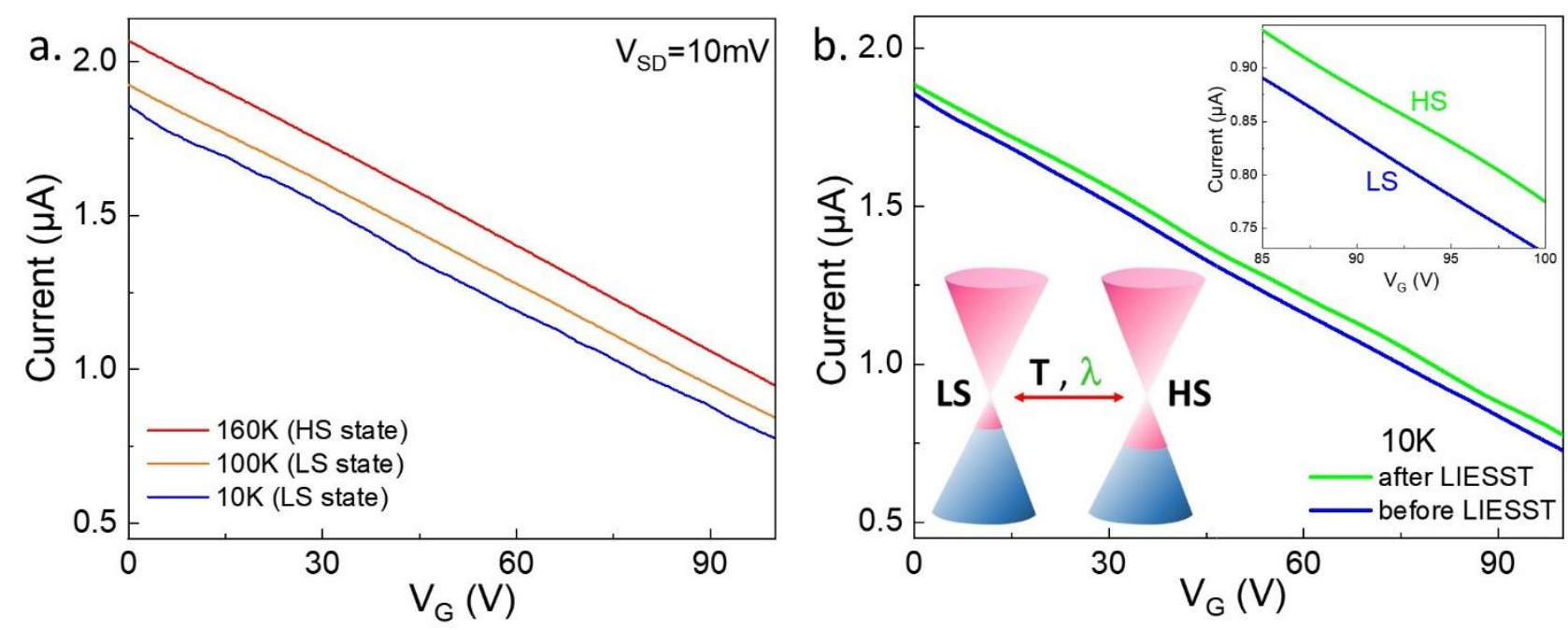

Figure 5. Transconductance measurement during Light-Induced Spin Transition and Thermal Spin transition. a. Current vs back gate voltage (VG) transfer curves measured on the SCO/graphene device at $10 \mathrm{~K}$ (in LS state), $100 \mathrm{~K}$ (in LS state) and $160 \mathrm{~K}$ (in HS state). b. Current vs. back gate voltage plot of the SCO/graphene device at $10 \mathrm{~K}$, performed in the dark, measured before (blue) and after (green) application of red light excitation $\left(\lambda_{1}=647 \mathrm{~nm}, 550 \mu \mathrm{W} . \mathrm{mm}^{-2}\right)$ during 1 hour. Upper inset highlights the shift between the LS and HS states. Lower inset illustrates the graphene band diagram and the change of holes concentration upon thermal and light induced spin transition.

\section{CONCLUSION}

Thin film SCO/graphene heterostructures combine the multi-stimuli responsivity of SCO molecules with the high conductivity and the ultimate surface sensitivity of the graphene monolayer. Its planar architecture allows an efficient opto-electrical detection of the spin transition for a better both thermal and optical control of the device. The principle of electrical detection of light-induced spin transition is uncovered, with isothermal partial reversibility, as well as the hysteretic bistable light imprint of a magnetic state of the molecular film. The scarcity of optical methods to store information in solids highlights the all-optical writing and erasing process of the spin state-related magnetic and conduction properties. Reducing the thickness of the SCO film and/or adding a spacer layer can help to better understand the coupling mechanism(s) involved in the change of graphene conduction resulting from the molecules switching, as well as possibly to shed light on the key issue of preserving the characteristics of SCO properties (cooperativity, bistability) when reducing the thickness down to the ultimate monolayer limit. ${ }^{[64]}$ While our work address the fundamental aspect of the LIESST effect in 
$\mathrm{SCO} / 2 \mathrm{D}$ heterostructures and consider long time switching mechanisms as proof of principle, relatively faster spin state switching (sub-ms time scale) can be envisioned while using nanosecond laser pulses within the thermal hysteresis loop of SCO nanoparticles. ${ }^{[71,72]}$ The electrical transparency of 2D materials including graphene can also be an advantage for imposing very large electric fields through substrate doping, ${ }^{[32,73]}$ and investigating how this impacts the SCO transition and their photoinduced bistability. ${ }^{[74,75]}$ This approach should unlock a stream of novel studies to revisit the physical mechanism of the photo-induced spin transition while investigating its coupling to the conduction properties of 2D materials. ${ }^{[28,76,77]}$ Because of the atomic-scale thickness of graphene and its exacerbated surface sensitivity, it is also a promising platform to explore the fast growing field of molecule/substrate interfacial properties, ${ }^{[78,79]}$ down to the single molecule level. ${ }^{[55,80]}$

\section{METHODS}

SCO synthesis and thin film deposition: the SCO compound was synthesized and sublimed $\left(1.5 \times 10^{-5}\right.$ mbar, $\left.117^{\circ} \mathrm{C}\right)$ with the conditions described in ref.[52] The vacuum deposition was simultaneously achieved on patterned graphene (the device), on a continuous graphene/ $\mathrm{SiO}_{2}$ and on $\mathrm{SiO}_{2} / \mathrm{Si}$ substrates. The thin films were annealed at $100^{\circ} \mathrm{C}$ for $1 \mathrm{~h} 30$ under primary vacuum.

Graphene device fabrication: Large area graphene devices, made of $300 \mu \mathrm{m}$ wide and $1.5 \mathrm{~mm}$ long stripes, are patterned into chemical vapor deposition (CVD) monolayer graphene transferred over $\mathrm{SiO}_{2}(300 \mathrm{~nm}) / \mathrm{Si}-\mathrm{p}+$ substrate (bought from Graphenea), using laser lithography (Heidelberg $\mu \mathrm{PG}$ 101) and Oxygen plasma (Plasma Prep III) processes. The substrate is firstly cleaned with acetone and rinsed with ethanol and isopropanol, before being dried by a nitrogen jet gas. AZ 1505 resist is then spin-coated at $4000 \mathrm{rpm}$ and baked at $100{ }^{\circ} \mathrm{C}$ for $50 \mathrm{~s}$. The well defined area on the substrate is exposed under UV Laser. The exposed resist is developed using a bath of AZ 726 MIF for $20 \mathrm{~s}$, before being rinsed in DI water. We then used Oxygen plasma etching ( $80 \mathrm{~s})$ to etch the graphene exposed 
area. Sample is then cleaned in acetone, isopropanol and dried in nitrogen jet gas. Similar laser lithography process is then used to form metallic interconnects. The metal deposition $(\mathrm{Ti}(7 \mathrm{~nm}) / \mathrm{Au}$ (43 nm) was carried out in Plassys MEB 550S, followed by lift-off in acetone, and similar cleaning, rinsing and drying of the substrate as detailed previously.

X-ray photoelectron spectroscopy (XPS): XPS measurements were performed on a K Alpha spectrometer from ThermoFisher, equipped with a monochromated X-ray Source $\left(\mathrm{Al} \mathrm{K} \mathrm{K}_{\alpha}, 1486.6 \mathrm{eV}\right)$ with a spot size of $400 \mu \mathrm{m}$. The hemispherical analyzer was operated in Constant Analyzer Energy (CAE) mode, with a pass energy of $200 \mathrm{eV}$ and a step of $1 \mathrm{eV}$ for the acquisition of surveys spectra, and a pass energy of $50 \mathrm{eV}$ and $20 \mathrm{eV}$ and a step of $0.1 \mathrm{eV}$ for the acquisition of narrow spectra. A "dual beam" flood gun was used to neutralize the charge build-up. The spectra obtained were treated by means of the CasaXPS software. A Shirley type background subtraction was used and the peak areas were normalized using the Scofield sensitivity factors. The binding energies were calibrated against the $\mathrm{C} 1 \mathrm{~s}$ binding energy set a $284.8 \mathrm{eV}$. The peaks were analyzed using mixed GaussianLorentzian curves ( $70 \%$ of Gaussian character).

Atomic Force Microscopy (AFM): The AFM imaging was carried out with a Innova SPM system in the tapping mode using TESPA-V2 tips (0.01-0.025 Ohm.cm Antimony (n) doped Si). The resonance frequency was set to $302 \mathrm{KHz}$. The resolution of the images is $1024 \times 1024$ with a scan rate of $0.1 \mathrm{~Hz}$.

Opto-electronic meas urement: Low-temperature electrical measurements were carried out in $\mathrm{He}$ flow cryostats of $1.5 \mathrm{~K}$ base temperature. A dual channel high precision source meter K2634B and a K2182A nanovoltmeter were used for DC measurements. Light excitation was sent down to the sample using an optical fiber based insert and an optical fiber mounted in the electronic measurement stick. We used a Coherent OBIS $647 \mathrm{~nm}$ LX laser for LIESST excitation and a Ti-Saphire laser in 
continuous wave regime $\left(850 \mathrm{~nm}, 100 \mu \mathrm{W} / \mathrm{mm}^{2}\right)$ as near-infrared source for reverse-LIESST excitation.

\section{ACKNOWLEDGMENT}

We thank Marc Ziegler, Olivier Cregut, and Mohamed Soliman for technical assistance in using the Ti-Saphir laser, and Fabien Chevrier for cryogenics. J.-F.D. acknowledges the financial support by MIXES (grant ANR-19-CE09-0028), COPIN (ANR-19-CE24-0022). This work is supported by a public grant overseen by the French National Research Agency (ANR) as part of the "Investissements d'Avenir" program (Labex NIE, ANR-11-LABX-0058, and ANR-10-IDEX-0002-02). The authors acknowledge the use of clean-room facilities from the STnano platform. A.S. and S.B. acknowledge support for the Indo-French Centre for the Promotion of Advanced Research(CEFIPRA). J.-F. D. and T.M. thank the Institut Universitaire de France (IUF) for financial support.

\section{CONTRIBUTION :}

J.-F. D., T. M. and B. D. conceived the project. N. K. fabricated the graphene device, with U. N. N. and H. M. providing technical assistance. N. K., J.-F. D. and B. K. built the photoconduction part of the transport measurement set-up. N. K. and J.-F. D. did the transport measurements, with expertise provided by M.-L. B. and T. M. for photoexcitation of SCO complex. A. T. synthesized the SCO complex and measured the AFM of the thin film. A. B. and A. T. deposited the SCO thin film. D. D. measured the XPS. A. S. and S. B. did the Raman measurements. M. L. performed XRD. N. K., M.L. B., B. D., T. M. and J.-F. D. analysed the data. J.-F. D. coordinated the writing of the paper, with T. M., M.-L. B. and B. D. All authors contributed to the discussions and the preparation of the manuscript. All the authors have given approval to the final version of the manuscript.

\section{SUPPORTING INFORMATION}


This material is available free of charge via the Internet at http://pubs.acs.org. It includes additional information regarding : 1. Raman spectroscopy; 2. Atomic Force Microscopy (AFM) imaging and profilometry; 3. X-ray powder diffraction; 4. X-ray Photoelectron Spectroscopy (XPS); 5. Reproducibility of thermal hysteresis conductance characteristics; 6. Conductance versus temperature characterization of graphene substrate without spin crossover material; 7.Photoconduction characterization of graphene/SCO heterostructure and graphene only devices; 8 . Successive conductance vs temperature cycles.

\section{AUTHOR INFORMATION}

Dayen Jean-Francois - Université de Strasbourg, CNRS, Institut de Physique et Chimie des

Matériaux de Strasbourg (IPCMS), UMR 7504, 23 rue du Loess, Strasbourg, 67034, France.

Institut Universitaire de France (IUF), 1 rue Descartes, 75231 Paris cedex 05, France.

dayen@unistra.fr

Mallah Talal - ENS Paris-Saclay, Université Paris-Saclay, 4 av. des Sciences, CS 30008, 91192 Gif sur Yvette, France. talal.mallah@universite-paris-saclay.fr

\section{Author Contributions}

The manuscript was written through contributions of all authors. All authors have given approval to the final version of the manuscript.

\section{Notes}

The authors declare no competing financial interest

\section{REFERENCES}

[1] S. Jan Van Der Molen, P. Liljeroth, J. Phys. Condens. Matter 2010, 22, 30.

[2] A. Bousseksou, G. Molnár, L. Salmon, W. Nicolazzi, Chem. Soc. Rev. 2011, 40, 3313.

[3] K. Senthil Kumar, M. Ruben, Coord. Chem. Rev. 2017, 346, 176.

[4] D. Pinkowicz, M. Rams, M. Mišek, K. V. Kamenev, H. Tomkowiak, A. Katrusiak, B. Sieklucka, J. Am. Chem. Soc. 2015, 137, 8795. 
[5] C. D. S. Brites, P. P. Lima, N. J. O. Silva, A. Millán, V. S. Amaral, F. Palacio, L. D. Carlos, Nanoscale 2012, 4, 4799.

[6] O. Sato, Nat. Chem. 2016, $8,644$.

[7] J. Ferrando-Soria, J. Vallejo, M. Castellano, J. Martínez-Lillo, E. Pardo, J. Cano, I. Castro, F. Lloret, R. Ruiz-García, M. Julve, Coord. Chem. Rev. 2017, 339, 17.

[8] S. I. Ohkoshi, K. Imoto, Y. Tsunobuchi, S. Takano, H. Tokoro, Nat. Chem. 2011, 3, 564.

[9] K. Szaciłowski, Chem. Rev. 2008, 108, 3481.

[10] P. Gütlich, Y. Garcia, in Ref. Modul. Chem. Mol. Sci. Chem. Eng., Elsevier, 2015.

[11] A. Cornia, P. Seneor, Nat. Mater. 2017, 16, 505.

[12] S. Sanvito, Chem. Soc. Rev. 2011, 40,3336.

[13] G. Molnár, S. Rat, L. Salmon, W. Nicolazzi, A. Bousseksou, Adv. Mater. 2018, 30, DOI 10.1002/adma.201703862.

[14] F. Prins, M. Monrabal-Capilla, E. A. Osorio, E. Coronado, H. S. J. Van Der Zant, Adv. Mater. 2011, 23, 1545 .

[15] J. Dugay, M. Giménez-Marqués, T. Kozlova, H. W. Zandbergen, E. Coronado, H. S. J. van der Zant, Adv. Mater. 2015, 27, 1288.

[16] A. Rotaru, J. Dugay, R. P. Tan, I. A. Guralskiy, L. Salmon, P. Demont, J. Carrey, G. Molnár, M. Respaud, A. Bousseksou, Adv. Mater. 2013, 25, 1745.

[17] A. Rotaru, I. A. Gural'skiy, G. Molnár, L. Salmon, P. Demont, A. Bousseksou, Chem. 
Commun. 2012, 48, 4163.

[18] Y. S. Koo, J. R. Galán-Mascarós, Adv. Mater. 2014, 26, 6785.

[19] S. Rat, M. Piedrahita-Bello, L. Salmon, G. Molnár, P. Demont, A. Bousseksou, Adv. Mater. 2018, 30, 1705275.

[20] E. J. Devid, P. N. Martinho, M. V. Kamalakar, I. Šalitroš, Ú. Prendergast, J. F. Dayen, V. Meded, T. Lemma, R. González-Prieto, F. Evers, T. E. Keyes, M. Ruben, B. Doudin, S. J. Van Der Molen, ACS Nano 2015, 9, 4496.

[21] Y. Murashima, M. R. Karim, N. Saigo, H. Takehira, R. Ohtani, M. Nakamura, M. Koinuma, L. F. Lindoy, K. Kuroiwa, S. Hayami, Inorg. Chem. Front. 2015, $2,886$.

[22] X. Zhang, Z. X. Wang, H. Xie, M. X. Li, T. J. Woods, K. R. Dunbar, Chem. Sci. 2016, 7, 1569.

[23] H. Banerjee, S. Chakraborty, T. Saha-Dasgupta, Chem. Mater. 2016, 28, 8379.

[24] P. S. Costa, G. Hao, A. T. N'Diaye, L. Routaboul, P. Braunstein, X. Zhang, J. Zhang, T. K. Ekanayaka, Q. Y. Shi, V. Schlegel, B. Doudin, A. Enders, P. A. Dowben, J. Phys. Condens. Matter 2020, 32, 034001.

[25] Y. Liu, N. O. Weiss, X. Duan, H.-C. Cheng, Y. Huang, X. Duan, Nat. Rev. Mater. 2016, 1, 16042.

[26] D. Jariwala, T. J. Marks, M. C. Hersam, Nat. Mater. 2017, 16, 170.

[27] F. Godel, L. D. N. Mouafo, G. Froehlicher, B. Doudin, S. Berciaud, Y. Henry, J.-F. Dayen, D. Halley, L. D. N. M. Florian Godel, F. Godel, L. D. N. Mouafo, G. Froehlicher, B. Doudin, S. Berciaud, Y. Henry, J.-F. Dayen, D. Halley, Adv. Mater. 2017, 29, 1604837. 
[28] M. Gobbi, E. Orgiu, P. Samorì, Adv. Mater. 2018, 30, 1706103.

[29] L. D. N. Mouafo, F. Godel, G. Melinte, S. Hajjar-Garreau, H. Majjad, B. Dlubak, O. Ersen, B. Doudin, L. Simon, P. Seneor, J. F. Dayen, Adv. Mater. 2018, DOI 10.1002/adma.201802478.

[30] C.-B. Huang, S. Witomska, A. Aliprandi, M.-A. Stoeckel, M. Bonini, A. Ciesielski, P. Samorì, Adv. Mater. 2019, 31, 1804600 .

[31] Y. Zhao, S. Bertolazzi, P. Samorì, ACS Nano 2019, 13, 4814.

[32] U. N. Noumbé, C. Gréboval, C. Livache, A. Chu, H. Majjad, L. E. Parra López, L. D. N. Mouafo, B. Doudin, S. Berciaud, J. Chaste, A. Ouerghi, E. Lhuillier, J.-F. Dayen, ACS Nano 2020, DOI 10.1021/acsnano.0c00103.

[33] G. Konstantatos, M. Badioli, L. Gaudreau, J. Osmond, M. Bernechea, F. P. G. de Arquer, F. Gatti, F. H. L. Koppens, Nat. Nanotechnol. 2012, 7, 363.

[34] D. Kufer, I. Nikitskiy, T. Lasanta, G. Navickaite, F. H. L. Koppens, G. Konstantatos, $A d v$. Mater. n.d., 27, 176.

[35] A. Allain, Z. Han, V. Bouchiat, Nat. Mater. 2012, 11, 590.

[36] K. Pi, K. M. McCreary, W. Bao, W. Han, Y. F. Chiang, Y. Li, S.-W. Tsai, C. N. Lau, R. K. Kawakami, Phys. Rev. B 2009, 80, 075406.

[37] G. Song, M. Ranjbar, D. R. Daughton, R. A. Kiehl, Nano Lett. 2019, 19, 7112.

[38] A. Mahmood, C. S. Yang, S. Jang, L. Routaboul, H. Chang, A. Ghisolfi, P. Braunstein, L. Bernard, T. Verduci, J. F. Dayen, P. Samorì, J. O. Lee, B. Doudin, Nanoscale 2019, 11, 19705.

[39] S. Parui, L. Pietrobon, D. Ciudad, S. Vélez, X. Sun, F. Casanova, P. Stoliar, L. E. Hueso, Adv. 
Funct. Mater. 2015, 25, 2972.

[40] M. I. Katsnelson, Mater. Today 2007, 10, 20.

[41] J. Dugay, M. Aarts, M. Giménez-Marqués, T. Kozlova, H. W. Zandbergen, E. Coronado, H. S. J. van der Zant, Nano Lett. 2017, 17, 186.

[42] E. P. Geest, K. Shakouri, W. Fu, V. Robert, V. Tudor, S. Bonnet, G. F. Schneider, Adv. Mater. 2020,32, 1903575.

[43] A. Hauser, in Top Curr Chem, Springer, Berlin, Heidelberg, 2012, pp. 155-198.

[44] C. Etrillard, V. Faramarzi, J.-F. Dayen, J.-F. Letard, B. Doudin, Chem. Commun. (Camb). 2011, 47, 9663 .

[45] C. Lefter, R. Tan, J. Dugay, S. Tricard, G. Molnár, L. Salmon, J. Carrey, A. Rotaru, A. Bousseksou, Phys. Chem. Chem. Phys. 2015, 17, 5151.

[46] C. Lefter, S. Rat, J. S. Costa, M. D. Manrique-Juárez, C. M. Quintero, L. Salmon, I. Séguy, T. Leichle, L. Nicu, P. Demont, A. Rotaru, G. Molnár, A. Bousseksou, Adv. Mater. 2016, 28, 7508.

[47] J. F. Létard, P. Guionneau, L. Rabardel, J. A. K. Howard, A. E. Goeta, D. Chasseau, O. Kahn, Inorg. Chem. 1998, 37, 4432.

[48] A. Desaix, O. Roubeau, J. Jeftic, J. G. Haasnoot, K. Boukheddaden, E. Codjovi, J. Linarès, M. Noguès, F. Varret, Eur. Phys. J. B 1998, 6, 183.

[49] F. Varret, K. Boukheddaden, E. Codjovi, C. Enachescu, J. Linarès, in Top Curr Chem, Springer, Berlin, Heidelberg, 2012, pp. 199-229. 
[50] M. Ruben, K. S. Kumar, Angew. Chemie Int. Ed. 2019, DOI 10.1002/anie.20191 1256.

[51] T. Mallah, M. Cavallini, Comptes Rendus Chim. 2018, 21, 1270.

[52] O. Iasco, M. L. Boillot, A. Bellec, R. Guillot, E. Rivière, S. Mazerat, S. Nowak, D. Morineau, A. Brosseau, F. Miserque, V. Repain, T. Mallah, J. Mater. Chem. C 2017, 5, 11067.

[53] X. Zhang, S. Mu, G. Chastanet, N. Daro, T. Palamarciuc, P. Rosa, J.-F. Létard, J. Liu, G. E. Sterbinsky, D. A. Arena, C. Etrillard, B. Kundys, B. Doudin, P. A. Dowben, J. Phys. Chem. C 2015, 119, 16293.

[54] J. F. Létard, J. Mater. Chem. 2006, 16, 2550.

[55] L. Zhang, Y. Tong, M. Kelai, A. Bellec, J. Lagoute, C. Chacon, Y. Girard, S. Rousset, M. Boillot, E. Rivière, T. Mallah, E. Otero, M. Arrio, P. Sainctavit, V. Repain, Angew. Chemie Int. Ed. 2020, anie. 202003896.

[56] G. Cao, X. Liu, Y. Zhang, W. Liu, M. Deng, G. Chen, G. Zhang, Q. Li, L. G. Beka, X. Li, X. Wang, ACS Appl. Mater. Interfaces 2019, 11, 12170.

[57] Y. D. Kim, M. H. Bae, J. T. Seo, Y. S. Kim, H. Kim, J. H. Lee, J. R. Ahn, S. W. Lee, S. H. Chun, Y. D. Park, ACS Nano 2013, 7, 5850.

[58] M. Buscema, J. O. Island, D. J. Groenendijk, S. I. Blanter, G. A. Steele, H. S. J. Van Der Zant, A. Castellanos-Gomez, Chem. Soc. Rev. 2015, 44,3691.

[59] T. Delgado, A. Tissot, C. Besnard, L. Guénée, P. Pattison, A. Hauser, Chem. - A Eur. J. 2015, 21,3664 .

[60] G. Chastanet, M. Lorenc, R. Bertoni, C. Desplanches, Comptes Rendus Chim. 2018, 21, 1075. 
[61] G. Chastanet, C. Desplanches, C. Baldé, P. Rosa, M. Marchivie, P. Guionneau, Chem. Squared 2018, DOI 10.28954/2018.csq.07.001.

[62] T. Delgado, C. Enachescu, A. Tissot, A. Hauser, L. Guénée, C. Besnard, J. Mater. Chem. C 2018, 6, 12698.

[63] C. Enachescu, R. Tanasa, A. Stancu, G. Chastanet, J. F. Látard, J. Linares, F. Varret, J. Appl. Phys. 2006, 99, 08J504.

[64] K. Bairagi, A. Bellec, C. Fourmental, O. Iasco, J. Lagoute, C. Chacon, Y. Girard, S. Rousset, F. Choueikani, E. Otero, P. Ohresser, P. Sainctavit, M. L. Boillot, T. Mallah, V. Repain, J. Phys. Chem. C 2018, 122, 727.

[65] F. Schedin, A. K. Geim, S. V. Morozov, E. W. Hill, P. Blake, M. I. Katsnelson, K. S. Novoselov, Nat. Mater. 2007, 6, 652.

[66] B. Guo, Q. Liu, E. Chen, H. Zhu, L. Fang, J. R. Gong, Nano Lett. 2010, 10, 4975.

[67] J. B. Bult, R. Crisp, C. L. Perkins, J. L. Blackburn, ACS Nano 2013, 7, 7251.

[68] S. Bonhommeau, T. Guillon, L. M. Lawson Daku, P. Demont, J. S. Costa, J. F. Létard, G. Molnár, A. Bousseksou, Angew. Chemie - Int. Ed. 2006, 45, 1625.

[69] A. Bousseksou, G. Molnár, P. Demont, J. Menegotto, J. Mater. Chem. 2003, 13, 2069.

[70] J.-P. Tuchagues, A. Bousseksou, G. Molnár, J. J. McGarvey, F. Varret, in Spin Crossover Transit. Met. Compd. III, Springer-Verlag, 2006, pp. 84-103.

[71] G. Gallé, C. Etrillard, J. Degert, F. Guillaume, J.-F. Létard, E. Freysz, Appl. Phys. Lett. n.d., DOI 10.1063/1.4792527. 
[72] E. Collet, L. Henry, L. Piñeiro-López, L. Toupet, J. Antonio Real, Curr. Inorg. Chem. 2016, 6, 61.

[73] U. N. Noumbé, C. Gréboval, C. Livache, T. Brule, B. Doudin, A. Ouerghi, E. Lhuillier, J. F. Dayen, Adv. Funct. Mater. 2019, DOI 10.1002/adfm.201902723.

[74] X. Zhang, P. S. Costa, J. Hooper, D. P. Miller, A. T. N'Diaye, S. Beniwal, X. Jiang, Y. Y in, P. Rosa, L. Routaboul, M. Gonidec, L. Poggini, P. Braunstein, B. Doudin, X. Xu, A. Enders, E. Zurek, P. A. Dowben, Adv. Mater. 2017, 29, n/a.

[75] X. Zhang, T. Palamarciuc, J. François Létard, P. Rosa, E. V. Lozada, F. Torres, L. G. Rosa, B. Doudin, P. A. Dowben, Chem. Commun. 2014, 50, 2255.

[76] Y. Zhao, S. Ippolito, P. Samorì, Adv. Opt. Mater. 2019, 7, 1900286.

[77] J. Sun, Y. Choi, Y. J. Choi, S. Kim, J. Park, S. Lee, J. H. Cho, Adv. Mater. 2019, 31, 1803831.

[78] J. F. Dayen, S. J. Ray, O. Karis, I. J. Vera-Marun, M. V. Kamalakar, Appl. Phys. Rev. 2020, 7, 011303.

[79] V. E. Campbell, M. Tonelli, I. Cimatti, J. B. Moussy, L. Tortech, Y. J. Dappe, E. Rivière, R. Guillot, S. Delprat, R. Mattana, P. Seneor, P. Ohresser, F. Choueikani, E. Otero, F. Koprowiak, V. G. Chilkuri, N. Suaud, N. Guihéry, A. Galtayries, F. Miserque, M. A. Arrio, P. Sainctavit, T. Mallah, Nat. Commun. 2016, 7, 1.

[80] K. Bairagi, O. Iasco, A. Bellec, A. Kartsev, D. Li, J. Lagoute, C. Chacon, Y. Girard, S. Rousset, F. Miserque, Y. J. Dappe, A. Smogunov, C. Barreteau, M. L. Boillot, T. Mallah, V. Repain, Nat. Commun. 2016, 7, 1. 
